\title{
Target deletion of the AAA ATPase PpCDC48II in Physcomitrella patens results in freezing sensitivity after cold acclimation
}

\author{
WANG Hui ${ }^{\dagger *}$, JIN Shuo ${ }^{\dagger}$, CHEN Xi, GEN XuKe \& HE YiKun \\ College of Life Science, Capital Normal University, Beijing 100037, China
}

Received July 21, 2011; accepted December 13, 2011

\begin{abstract}
CDC48 is a highly conserved protein in eukaryotes and belongs to the AAA (ATPase associated with a variety of cellular activities) superfamily. It can interact with many different co-factors and form protein complexes that play important roles in various cellular processes. According to the Physcomitrella patens database, one member of the ATPases, the cell cycle gene PpCDC48II, was cloned. PpCDC48II contains two typical ATPase modules and is highly homologous to AtCDC48A. $P p C D C 48 I I$ was up-regulated in mRNA levels after incubation at $0^{\circ} \mathrm{C}$ for 36 and $72 \mathrm{~h}$. To further elucidate protein function, we disrupted the PpCDC48II gene by transforming $P$. patens with the corresponding linear genomic sequences. When treated to the same freezing stress, it was found that $P p C D C 48 I I$ knockout plants were less resistant to freezing treatment than wild type after acclimation. This suggested that $P p C D C 48 I I$ was an essential gene for low-temperature-induced freezing tolerance in P. patens cells.
\end{abstract}

PpCDC48II, Physcomitrella patens, gene knockout, cold acclimation, freezing tolerance

Citation: Wang H, Jin S, Chen X, et al. Target deletion of the AAA ATPase PpCDC48II in Physcomitrella patens results in freezing sensitivity after cold acclimation. Sci China Life Sci, 2012, 55: 150-157, doi: 10.1007/s11427-012-4277-z

Physcomitrella patens represents an excellent system to study gene function because the generation of targeted knockout plants is facilitated by high homologous recombination frequency $[1,2]$. In addition, $P$. patens was originally chosen to study plant differentiation processes, which were facilitated by the haploid status of the moss protonema. The moss $P$. patens has become accessible to state-of-the-art molecular and genetic analyses, which have promoted the system for analyzing almost all aspects of plant biology.

Low temperature is considered one of the most important environmental factors that limit growth, development, and distribution of plants. However, some temperate zone plants can adapt to the onset of low temperatures by producing chemical and physiological metabolites during late autumn and early spring, thus improving their resistance to low

$\lceil$ Contributed equally to this work

*Corresponding author (email: huiwangl@sina.com) temperature during winter. This indicates that plants evolve a set of perfect mechanisms to respond to low temperature.

Some results demonstrated that cold treatment clearly enhanced freezing tolerance in $P$. patens protonema cells. $P$. patens treated at low temperatures exhibited not only an increased tolerance to freezing but also higher expression levels of genes present in cold-treated cells [3]. Therefore, elucidating the intracellular functions of cold-regulated genes is important for understanding cold acclimation mechanism.

In a recent study, we used two powerful and reproducible PCR-based differential screening techniques, cDNA amplified fragment length polymorphism (cDNA-AFLP) and suppression subtractive hybridization ( $\mathrm{SSH}$ ), to isolate differentially expressed ESTs during cold acclimation in $P$. patens [4]. Of the 768 independent clones from the SSH cDNA library, approximately $50.9 \%$ were differentially regulated during cold acclimation. We identified 201 up- 
regulated, and 190 down-regulated clones using subtracted PCR product probes. These clones were then sequenced for further analysis. In all, we sequenced 420 EST clones from the two libraries for further analysis. According to EST analysis, we found that cold acclimation resulted in changes in $P p C D C 48$ genes expression levels. We further confirmed that $P p C D C 48 I I$ displayed up-regulated mRNA levels after incubation at $0^{\circ} \mathrm{C}$ for 36 and $72 \mathrm{~h}$ using RT-PCR.

According to these results, we cloned PpCDC48II from the $P$. patens. The gene contains two typical ATPase modules and is highly homologous to AtCDC48A. CDC48 is a member of the AAA (ATPases associated with various cellular activities) protein family. In zebrafish cells, cold-inducible expression of the cell cycle gene CDC48 promoted cell proliferation during cold acclimation [5]. In addition, yeast CDC48 has been implicated in a plethora of functions that include cell-cycle regulation, membrane fusion, stress response, and endoplasmic reticulum (ER)-associated degradation (ERAD) [6]. Its highly conserved mammalian counterpart, p97, has been further implicated in the reformation of the nucleus [7], organelle biogenesis [8], myofibril organization [9], and protein degradation of Hif1-a [10], and HMG-CoA reductase [11]. Two underlying properties of Cdc48/p97 contribute to its myriad functions: its ATPase activity and the ability to bind ubiquitin (Ub) [12].

Compared to the extensive studies of CDC48 homologs in yeasts and mammals, the understanding of CDC48 in higher plants is rudimentary. The Arabidopsis genome encodes three CDC48 isoforms: AtCDC48A (At3g09840), $A t C D C 48 B$ (At3g53230), and AtCDC48C (At5g03340) [13]. These isoforms are predicted to share 91\% (AtCDC48B) and 95\% (AtCDC48C) amino acid identity with AtCDC48A [14]. Expression of AtCDC48A mRNA is highest in proliferating cells of the vegetative shoot, the root, and the flowers in rapidly growing plants [15-17]. Using immunofluorescence microscopy, AtCDC48A has been shown to be localized to the cytoplasm, the nucleus, and the phragmoplast mid zone during cytokinesis $[14,15]$. Loss-of-function and dominant negative mutant studies indicated multiple roles for AtCDC48 in cell division, growth, and development in Arabidopsis [18]. At the subcellular level, $\mathrm{NgCDC} 48$, a tobacco CDC48 homolog, resided in the endoplasmic reticulum (ER) of protoplasts. Transgenic tobacco plants (35S:anti- $\mathrm{NgCDC48}$ ), in which the $\mathrm{NgCDC48}$ gene was suppressed using antisense RNA method, exhibited severely aberrant development of both vegetative and reproductive organs, resulting in arrested shoot and leaf growth, and sterile flowers. These results indicate the tantalizing possibility that CDC48-mediated degradation of an as-yet unidentified protein(s) in the ER might be a critical step for cell growth and expansion in tobacco leaves [19].

We knocked out the PpCDC48II gene through homologous recombination. The mutant plants were less resistant to freezing stress than wild type after acclimation. It is suggested that cold-induced expression of PpCDC48II is essen- tial for low-temperature-induced freezing tolerance in $P$. patens cells.

\section{Material and methods}

\subsection{Plant material and growth condition}

The moss $P$. patens was maintained axenically in $\mathrm{PPNH}_{4}$ medium as described previously [20], supplemented with $7 \mathrm{~g} \mathrm{~L}^{-1}$ agar when required. Plants were grown either in biofermenters or in jars in a phyto-chamber with a discontinuous white light cycle $\left(16 \mathrm{~h}\right.$ light at $25^{\circ} \mathrm{C} / 8 \mathrm{~h}$ dark at $18^{\circ} \mathrm{C}$ ) at quantum irradiance of $200 \mu \mathrm{mol} \mathrm{m} \mathrm{m}^{-2} \mathrm{~s}^{-1}$. Moss cultures were propagated by fragmenting old cultures in sterile water and transferring aliquots of this suspension to fresh medium.

\subsection{RNA extraction and cDNA synthesis}

Total RNA was obtained from two-week-old gametophores using a phenol-based extraction method. Poly(A) RNA was isolated from total RNA using the Qiagen Oligotex mRNA Kit (Qiagen, Germany). For RT-PCR analyses, cDNA was synthesized using an RNA PCR Kit (Takara, Japan).

\subsection{Recombinant DNA techniques}

Manipulation of nucleic acids was performed using standard protocols or, when appropriate, according to the manufacturer's instructions. DNA sequencing was performed in an automated ABI PRISM 3100 DNA sequencer (Applied Biosystems, http://www.appliedbiosystems.com). PCR amplifications of $P p C D C 48 I I$ cDNA fragments were carried out on double-stranded cDNA synthesized from total RNA using the cDNA Synthesis System Kit (Takara, Japan) and primers: RTII-f: 5'-ATTCTAGAATGGCGACTCCCGCAG3' (PpCDC48II forward) and RTII-r: 5'-GCCGTCGACGCTATACAAATCATCA-3' (PpCDC48II reverse) designed using EST sequences identified in databases. The resulting PCR fragments were cloned into the pGM-T Vector Cloning Kit (Takara Japan).

\subsection{Semi-quantitative RT-PCR (SQRT-PCR) analysis}

For cDNA synthesis, $1 \mu \mathrm{g}$ of total RNA was first treated with DNase I (Sigma-Aldrich, USA) for $15 \mathrm{~min}$ at room temperature, and then the enzyme was inactivated by heating at $70^{\circ} \mathrm{C}$ for $10 \mathrm{~min}$. Reverse transcription was performed with the cDNA Synthesis System Kit (Takara, Japan). Synthesized cDNAs were purified using the Gel Extraction Kit. Semi-quantitative RT-PCR analysis for $P p C D C 48 I I$ cold-inducible expression was performed using $1 \mu \mathrm{L}$ of the cDNA, and ExTaq polymerase with the supplied buffer and dNTP (Takara-Bio, Japan). The PCR conditions 
were as follows: 30 (PpCDC48II) or 26 (Ppactin3) cycles at $95^{\circ} \mathrm{C}$ for $30 \mathrm{~s}, 59^{\circ} \mathrm{C}(P p C D C 48 I I)$ or $55^{\circ} \mathrm{C}$ (Ppactin3) for $30 \mathrm{~s}$, and $72^{\circ} \mathrm{C}$ for $15 \mathrm{~s}$. A $5 \mu \mathrm{L}$ aliquot of each PCR reaction was separated on an agarose gel. We performed SQRT-PCR analysis with primers 5'-ATGGCGACTCCCGCAGCTGAA-3' and 5'-AAACGGAAGAACATGAATTC-3' for PpCDC48II.

\subsection{Generation of the PpCDC48II knockout lines}

For the PpCDC48II knockout construct, genomic DNA fragments $(1565 \mathrm{bp})$ were amplified using the primers 5'GCTTATCTAAAACTCGGAAC-3' (forward) and 5'-CAAATCATCATCATCAGCTGCA-3' (reverse). DNA fragments were cloned into the pGMT-T vector (Takara Japan). The neomycin phosphotransferase ( $N p t$ II) selection cassette was amplified from pTN182 vector using Hind III and BsS HII restriction sites. The plasmids containing the PpCDC48II fragment were digested with Hind III and Bss HII and the excised selection cassette was inserted to yield the PpCDC48II knockout constructs. For transfection, 30 $\mu \mathrm{g}$ of the knockout constructs was amplified by PCR, producing a linear fragment that contained the Npt II selection cassette flanked by $P p C D C 48 I I$ genomic sequences. Stable antibiotic-resistant clones were selected after two rounds of incubation in PPNH4 medium supplemented with $30 \mu \mathrm{g}$ $\mathrm{mL}^{-1}$ of G418 (Sigma-Aldrich Chemie, http://www.sigmaaldrich.com). The first screening of putative mutant plants was carried out using three independent PCR reactions on genomic DNA purified from transformant plants. The first reaction spanned the complete targeted regions using the primers $\mathrm{P}_{1 \mathrm{f}}$ : 5'-GCTTATCTAAAACTCGGAAC-3' and $\mathrm{P}_{1 \mathrm{r}}$ : 5'-CAAATCATCATCATCAGCTGCA-3'. The second reaction amplified part of the marker cassette using primers Nfwd: 5'-CACTATCCTTCGCAAGACCCTTCCTC-3' and Nrev: 5'-ATCCTTCCTTGTCTTCCTCCTTCCTTCCGT-3'. The final reactions amplified part of the $5^{\prime}$ and $3^{\prime}$ gene regions outside the knockout construct using the primers $\mathrm{P}_{2 \mathrm{f}}$ : 5'-TATGTTTTGGTTTTTGACGTG-3', $\mathrm{P}_{2 \mathrm{r}}$ : 5'-TGAGGAGCCACCTTCCTTTTC-3', $\mathrm{P}_{3 \mathrm{f}}$ : 5'-TTTTGTTCCCTTTAGTGAGGGT-3' and $\mathrm{P}_{3 \mathrm{r}}$ : 5'AAGCAGCTCAATTCATTCACAG-3'. These amplifications produced the expected fragments. The basic defects of Ppcdc48II mutants were studied in two independent lines. For the expression analysis of Ppcdc48II plants, $1 \mu \mathrm{g}$ of total RNA from wild type and Ppcdc48II protonema were used. Reverse transcription was done according to the manufacturer's protocol. RT-PCR was performed to confirm the PpCDC48II knockout.

\subsection{Freezing tests}

For freezing tests, two-week-old gametophores cold-acclimated at $0^{\circ} \mathrm{C}$ for $0,12,24,48$, and $72 \mathrm{~h}$ were frozen for 90 min at $-6,-4$, and $-10^{\circ} \mathrm{C}$, and then incubated overnight in the dark at $4^{\circ} \mathrm{C}$. The next day, gametophores were placed in fresh medium and incubated at $25^{\circ} \mathrm{C}$ under a $16 \mathrm{~h} \mathrm{light} / 8 \mathrm{~h}$ dark cycle for $2 \mathrm{~d}$ to observe the phenotype and $10 \mathrm{~d}$ to calculate survival efficiency.

\subsection{Measurement of freezing tolerance}

Two-week-old gametophores tissues of wild type and mutant Ppcdc48II were treated for freezing tests. Freezing tolerance was determined by measuring electrolyte leakage from cells, as previously described [21], and the survival efficiency.

\subsection{Measurement of gametophores chlorophyll con- tents}

Two-week-old gametophores tissues treated for freezing tests were harvested and chlorophyll was extracted from each colony using dimethylformamide. Chlorophyll contents (Chl a+Chl b=17.67A646.8+7.12A663.8) were measured using a spectrophotometer, and calculated according to previous description [22]. Chlorophyll contents were conventionally used for growth quantification of moss.

\section{Results}

\subsection{Isolation and characterization of $C D C 48$-like genes in Physcomitrella}

We searched for genes encoding CDC48 in the $P$. patens EST database [23], as well as in the genome database (http://genome.jgi-psf.org/Phypa1_1/Phypa1_1.home.html), and identified six genes containing two highly conserved ATPase domains. The number of $C D C 48$-like genes is more than that of Arabidopsis. Among them, we cloned the PpCDC48II gene. The coding region of full-length Physcomitrella PpCDC48II cDNA is 2448 bp long, which encode a 816 amino acid protein with a predicted molecular mass of $90.4 \mathrm{kD}$, similar to that of yeast $(92.0 \mathrm{kD})$, human (89.3 kD), and Arabidopsis (89.4-90.4 kD) CDC48 proteins (Figure 1A). As in other CDC48 homologs, the PpCDC48II protein contains two conserved ATPase domains, in which the Walker A and Walker B motifs are well conserved. Multiple alignments of the deduced PpCDC48 amino acid sequence with those of previously identified CDC48 proteins are shown in Figure 1B. PpCDC48II is identical to the flowering plant CDC48 proteins in Arabidopsis (88\%), rice $(89 \%)$, and tobacco $(89 \%)$. Furthermore, PpCDC48II shares a significant degree of sequence identity with yeast (67\%) and human $(78 \%)$ proteins (Figure $1 \mathrm{~A}$ and $\mathrm{B})$. Therefore, the isolated cDNA clone was found to encode Physcomitrella homologs of Arabidopsis AtCDC48. 

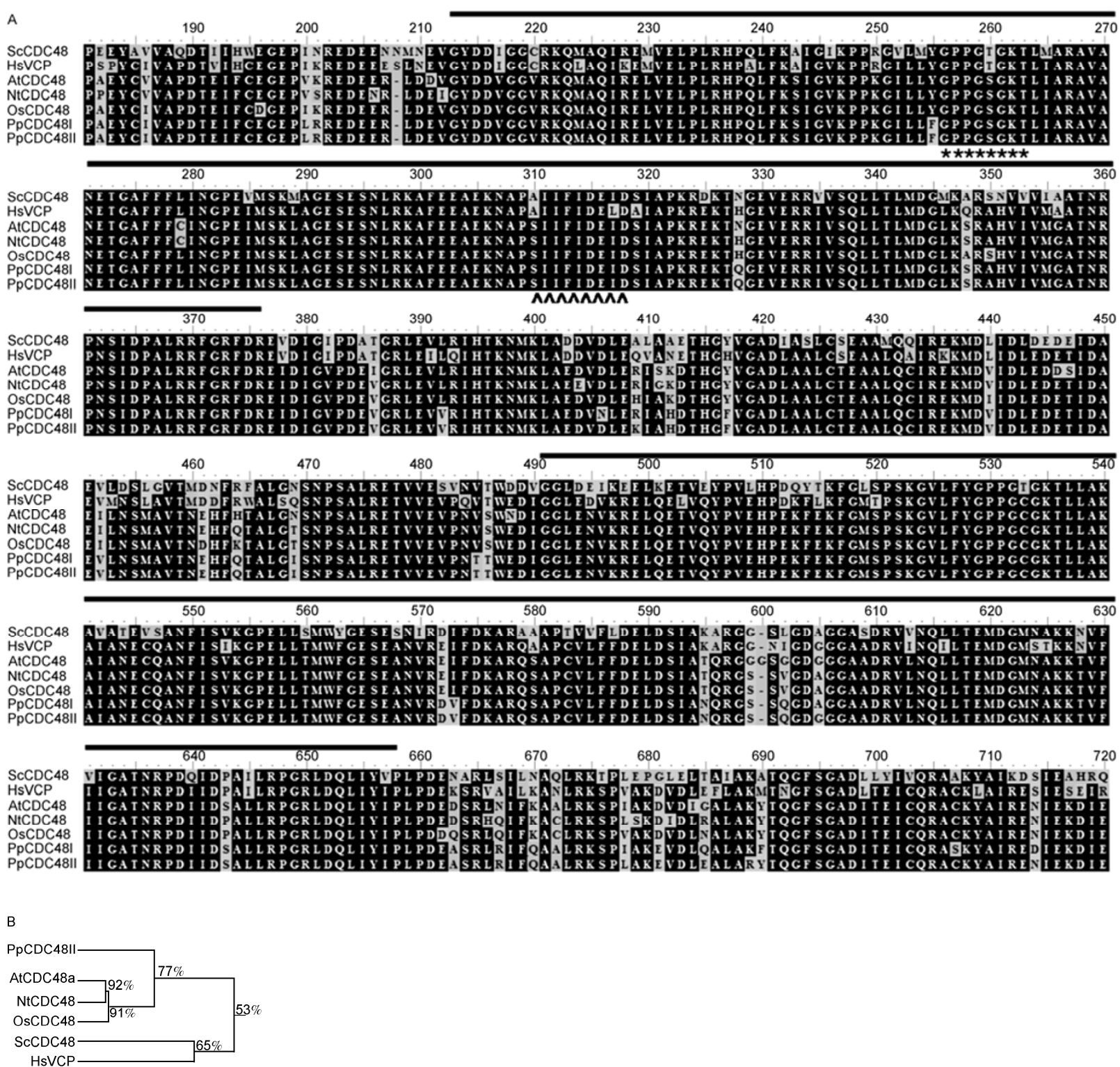

Figure 1 Sequence analysis of Physcomitrella PpCDC48II. A, Comparison of the deduced amino acid sequence of Physcomitrella PpCDC48II with CDC48 homologs from Arabidopsis (AtCDC48a), rice (OsCDC48), yeast (ScCDC48), tobacco (NtCDC48), and human (HsVCP). Amino acids that are identical in all seven proteins are shown in the same color. Solid lines represent two highly conserved AAA cassette domains. The Walker A and Walker B motifs are indicated by asterisk and arrowheads, respectively. Dashes show gaps introduced into the amino acid sequences for optimal sequence alignment. B, Phylogenetic analysis of CDC48 proteins from tobacco (NtCDC48), Arabidopsis (AtCDC48a), rice (OsCDC48), yeast (ScCDC48p), and human (HsVCP). The dendrogram was constructed using DNAMAN software with the neighbor-joining method. The tree is drawn to scale, with branch lengths representing units of evolutionary distance.

\subsection{Targeted disruption of $P p C D C 48 I I$ caused de-} creased freezing tolerance

To confirm $P p C D C 48 I I$ function, we performed a loss-offunction analysis and disrupted $P P C D C 48 I I$ via homologous recombination (Figure 2A). Two independent disruptants (lines 2 and 3) were selected after PCR analysis confirmed the complete deletion of the PpCDC48II coding sequence (Figure 2B). In addition, RT-PCR analysis confirmed that both transgenic lines lost $P p C D C 48 I I$ expression while retaining $P p C D C 48 I I$ expression in the wild type (Figure 2C).
To elucidate that it is low temperature itself that enhanced freezing tolerance in gametophytes, we treated the wild type and mutant at $-6^{\circ} \mathrm{C}$ for various time courses. Survival of the freeze-thawed cells was determined by measuring electrolyte leakage (Figure 3B). The results indicated both the wild type and the mutant gametophytes were freezing hypersensitive. We next evaluated freezing sensitivities of wild type and disruptants after cold acclimation at $0^{\circ} \mathrm{C}$. The gametophores grown at $25^{\circ} \mathrm{C}$ for $14 \mathrm{~d}$ were transferred to fresh agar plates and incubated at $0^{\circ} \mathrm{C}$ for $0,12,48$, and $72 \mathrm{~h}$. The cold 
acclimated gametophores, including wild type and Ppcdc48II mutants, were frozen at $-6^{\circ} \mathrm{C}$ for $90 \mathrm{~min}$ and thawed at $4{ }^{\circ} \mathrm{C}$ overnight, and then the treated plants were transferred to normal condition for $2 \mathrm{~d}$. Freezing tolerance of $P$. patens gametophores was examined by measuring electrolyte leakage after treatment (Figure 3E). The results indicated that incubation temperature had differential effects on the freezing tolerance of wild type and mutant gametophores (Figure 3C). Most wild type gametophores cold acclimated for $48 \mathrm{~h}$ at $0^{\circ} \mathrm{C}$ increased freezing tolerance and survived. In contrast, the survival of Ppcdc48II plants markedly decreased even with cold acclimation for $72 \mathrm{~h}$. The $P$. patens gametophores freezing tolerance during different temperature after $72 \mathrm{~h}$ cold acclimation is shown in Figure $3 \mathrm{~F}$ and Table 1. Both wild type and Ppcdc48II acclimated at $0^{\circ} \mathrm{C}$ for $72 \mathrm{~h}$ hardly survived at $-10^{\circ} \mathrm{C}$. About $70 \%$ of acclimated wild type gametophores can survive at $-6^{\circ} \mathrm{C}$, while the survival efficiency of mutants was only $10 \%$. In addition, the growth of the frozen-thawed gametophores was also evaluated by their chlorophyll content, which is conventionally used for growth quantification of moss (Figure 3D). The results were consistent with the freezing tests. The data demonstrated that disruption of PpCDC48II led to freezing-hypersensitivity after cold acclimation, providing concrete evidence for the role of PpCDC48II as a positive regulator of cold-induced freezing tolerance in moss. The data also suggested that the acquisition of freezing tolerance in $P$. patens involved a
PpCDC48II-mediated signaling pathway.

\subsection{Expression of $P p C D C 48 I I$ after cold-treatment}

It is well documented that CDC48 proteins participated in multiple cellular processes. We examined the expression of $P p C D C 48 I I$ gene during cold treatment in $P$. patens (Figure $3 \mathrm{~A})$. The results indicated that the PpCDC48II gene encoding AAA ATPase was regulated by low temperatures. The expression level of $P p C D C 48 I I$ was slightly up-regulated at $24 \mathrm{~h}$, distinctly up-regulated at $36 \mathrm{~h}$, and peaked at $72 \mathrm{~h}$ after incubation at $0^{\circ} \mathrm{C}$, a cold acclimated temperature.

\section{Discussion}

Gene inactivation by targeted gene replacement or by RNA interference, together with the nearly completed sequencing of the Physcomitrella patens genome, opens the way for detailed functional study of genes involved in both development and metabolism.

It is known that low-temperature treatment causes various biochemical and physiological changes in plant cells, including expression of numerous genes, accumulation of various proteins, increase of antioxidants, and activity of various metabolic enzymes [24-27]. Many results demonstrated that cold-induced, highly hydrophilic proteins such as LEA proteins play important roles in the enhancement of
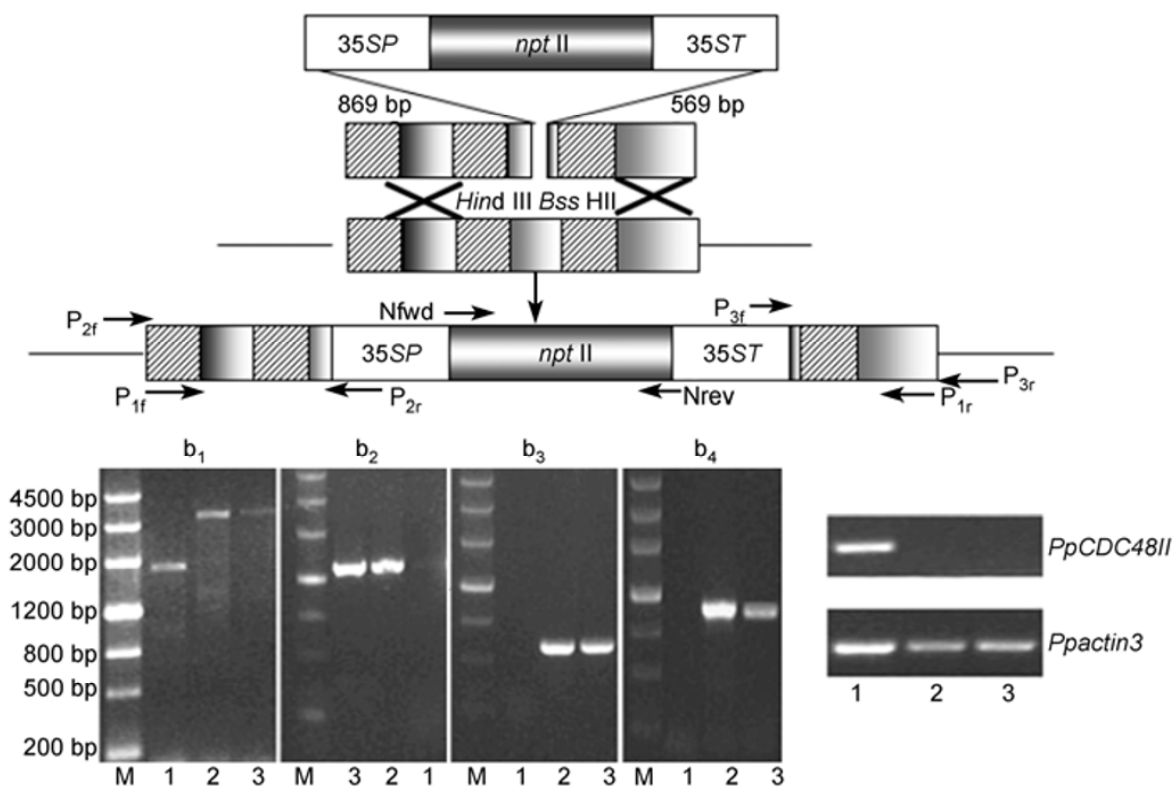

Figure 2 Generation and molecular analysis of PpCDC48II knockout lines. A, Insertion of a Npt II selection marker cassette into Hind III and Bss HII restriction site in exon 7 of the $P p C D C 48 I I$ gene (top); the resulting PpCDC48II knockout construct (middle); genomic structure after integration of the PpCDC48II knockout construct via homologous recombination (bottom). Primers used for the analysis of transgenic lines are indicated by arrows and Roman numerals. Dark gray box, Npt II cassette; boxes with fine lines, exon sequences within the knockout construct; gray boxes, introns flanking the knockout construct. B, PCR analysis to confirm 5' and $3^{\prime}$ integration of the PpCDC48II knockout construct using primer $\mathrm{P}_{1 \mathrm{f}}$ and $\mathrm{P}_{1 \mathrm{r}}\left(\mathrm{b}_{1}\right)$; $N p t$ II identification using primer Nfwd and Nrev $\left(b_{2}\right) ; 5^{\prime}$ integration analysis using primers $\mathrm{P}_{2 \mathrm{f}}$ and $\mathrm{P}_{2 \mathrm{r}}\left(\mathrm{b}_{4}\right) ; 3^{\prime}$ integration analysis using primers $\mathrm{P}_{3 \mathrm{f}}$ and $\mathrm{P}_{3 \mathrm{r}}\left(\mathrm{b}_{3}\right)$; $\mathrm{PCR}$ products obtained from wild type line 1 and Ppcdc48II mutant line 3 and line 2. C, RT-PCR analysis using primers RTII-f and RTII-r (PpCDC48II) and primers for the control gene Ppactin3. 1, wild type; 2 and 3, Ppcdc48II knockout line 2 and line 3. 
A
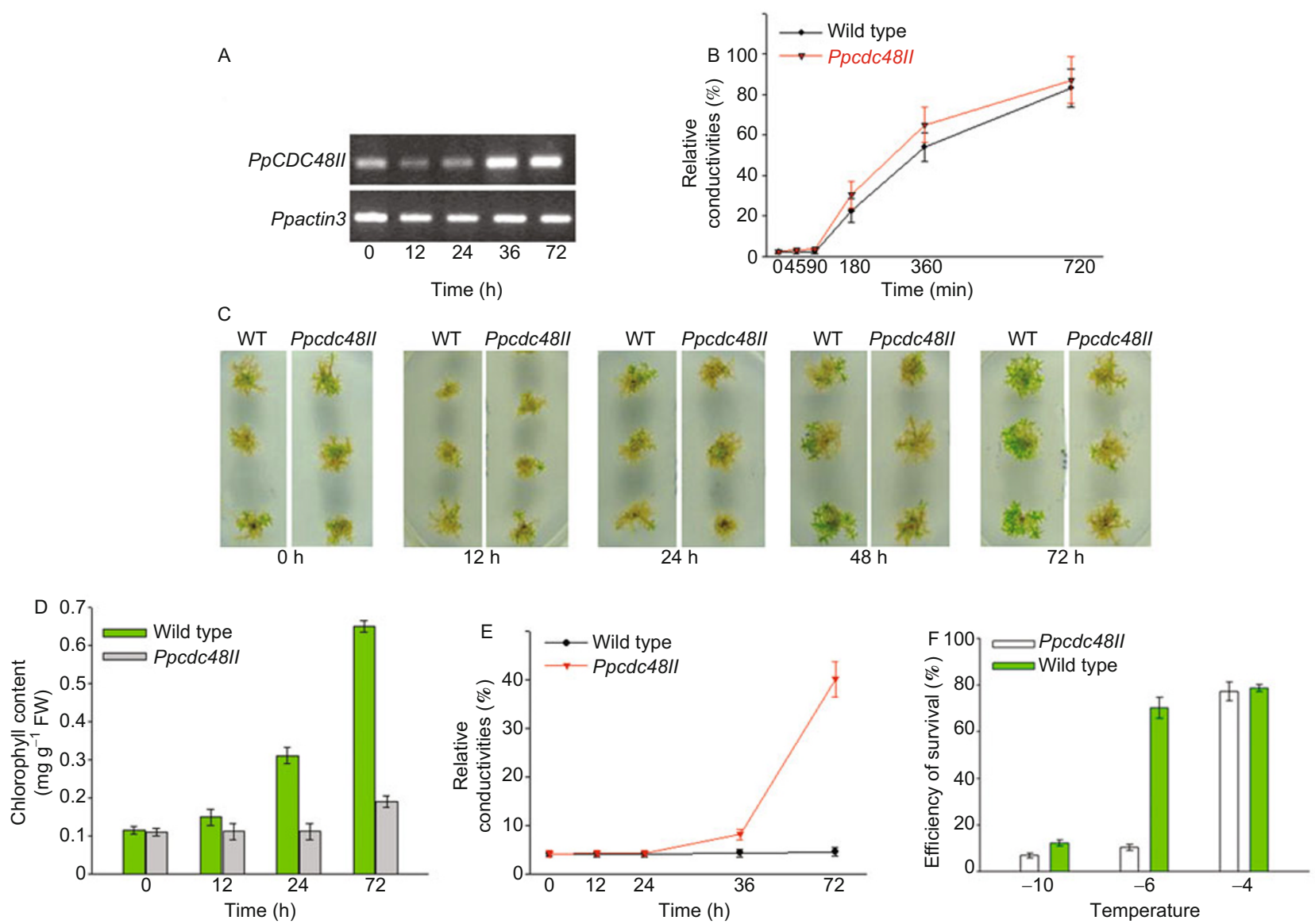

Figure $3 P p c d c 48 I I$ mutant lines were more susceptible to freezing stress after cold acclimation. A, Expression of $P p C D C 48 I I$ during cold acclimation. Relative PpCDC48II mRNA expression was analyzed using SQRT-PCR. RNAs were isolated from P. patens gametophores that were cold-acclimated at $0^{\circ} \mathrm{C}$ for 12, 24, 36, and $72 \mathrm{~h}$. Ppactin 3 was used as an endogenous control. B, Time course changes of P. patens gametophores freezing tolerance after treatment at $-6^{\circ} \mathrm{C}$. Two-week-old gametophores tissues were incubated on $\mathrm{PPNH}_{4}$ medium at $-6^{\circ} \mathrm{C}$ for various time points. Cell survival was determined by measuring electrolyte leakage after freeze-thawing. Error bars indicate standard errors of three independent experiments. C, The freezing sensitivity of Ppcdc48II mutants after cold acclimation. D, Measurement of gametophores chlorophyll contents after freezing tests. E, Time course of cold-induced freezing tolerance of wild type and $P p c d c 48 I I$ gametophore cells after acclimation. F, The survival efficiency after $10 \mathrm{~d}$ of refreshing growth. Two-week-old wild type and Ppcdc48II gametophores were incubated on $\mathrm{PPNH}_{4}$ medium at $0^{\circ} \mathrm{C}$ for the indicated time periods and subjected to equilibrium freezing to $-6^{\circ} \mathrm{C}$ for $90 \mathrm{~min}$. After thawing, the tissues were grown on fresh $\mathrm{PPNH}_{4}$ medium at $23^{\circ} \mathrm{C}$ for $2 \mathrm{~d}$. The growth quantification was determined by measuring gametophores chlorophyll content (D), and survival was determined by measuring electrolyte leakage after thawing (E). Two-week-old wild type and Ppcdc48II gametophores that were acclimated at $0^{\circ} \mathrm{C}$ for $72 \mathrm{~h}$, were frozen at $-4,-6$, and $-10^{\circ} \mathrm{C}$ for $90 \mathrm{~min}$. After thawing, the tissues were grown on fresh $\mathrm{PPNH}_{4}$ medium at $23^{\circ} \mathrm{C}$ for $10 \mathrm{~d}$. The survival efficiency was calculated $(\mathrm{F})$, and the values are shown in Table $1(N=50)$.

Table 1 Freezing tolerance of $P$. patens gametophores after cold acclimation ${ }^{\text {a) }}$

\begin{tabular}{|c|c|c|c|c|}
\hline \multicolumn{3}{|c|}{ Ppcdc48II } & \multicolumn{2}{|c|}{ Wild type } \\
\hline & Survival efficiency $(\%)^{\#}$ & SD & Survival efficiency $(\%)^{\#}$ & $\mathrm{SD}$ \\
\hline$-4^{\circ} \mathrm{C}$ & 77.30 & 0.042 & 78.6 & 0.014 \\
\hline$-6^{\circ} \mathrm{C}$ & 10.32 & 0.015 & 70.27 & 0.045 \\
\hline$-10^{\circ} \mathrm{C}$ & 6.91 & 0.011 & 12.31 & 0.013 \\
\hline
\end{tabular}

a) SD, standard deviation. \#, this test was repeated three times. For each test, the number of plants used in each line was greater than 50 .

freezing tolerance after low-temperature treatments in $P$. patens [28], as reported in higher plants [29,30]. Therefore, elucidating the intracellular functions of cold-regulated genes is important for understanding the cold acclimation mechanism.

One cold-inducible protein was first identified in Physcomitrella. Like other CDC48 homologs, PpCDC48II contained two highly conserved AAA-ATPase domains (Figure 1A), including the Walker A and B motifs, which were remarkably homologous not only to plant proteins, but also to yeast and human proteins. Therefore, because of the high degree of homology in amino acid sequence and domain structure, the cloned protein named PpCDC48II is the Physcomitrella homolog of Arabidopsis AtCDC48s. 
The N-terminal region of Physcomitrella PpCDC48II contains two putative nuclear localization signals (KKDFSTAILERKK and KGKKRKD), which are important in CDC48 protein translocation into the nucleus [15]. Amino acid sequence analysis indicated that PpCDC48II lacked membrane-spanning regions and was a soluble protein. These sequence characteristics indicated a tantalizing possibility that PpCDC48II localized to the nucleoplasm and the cytoplasm, as do AtCDC48 and ScCDC48p [31,32], may be transported between the nucleus and cytoplasm, and function in both places.

The AAA-ATPase CDC48/p97 in non-plant systems has been shown to function in various pathways. However, the cellular roles of plant CDC48 proteins are rudimental. In Arabidopsis, localization studies and other studies implicated a role for AtCDC48 in plant cytokinesis, cell expansion and division. The independent Arabidopsis T-DNA insertion alleles of Atcdc48A were recessive and displayed the same phenotypes, including defects in pollen tube germination and expansion embryo developmental arrest, and seeding lethality [18]. The tobacco NgCDC48, located in the endoplasmic reticulum (ER), was recently implicated in ER-associated degradation of an as-yet unidentified target protein(s) [19].

To confirm PpCDC48II function under cold conditions, we constructed the transgenic Physcomitrella plants in which $P p C D C 48 I I$ was knocked out through homologous recombination (Figure 2). Slow equilibrium freezing of $P$. patens gametophores grown under normal growth conditions to $-6^{\circ} \mathrm{C}$ killed more than $90 \%$ of the cells, indicating that the gametophore cells are freezing-sensitive (Figure 3B). Cold treatment for $72 \mathrm{~h}$ dramatically increased the freezing tolerance of wild type gametophores, while the Ppcdc48II mutants failed to generate freezing tolerance after cold acclimation, which indicated PpCDC48II plays important roles in the enhancement of freezing tolerance during cold acclimation. In addition, cold-treatment resulted in a remarkable increase in $P p C D C 48 I I$ gene expression within $72 \mathrm{~h}$ (Figure 3A). These results suggested that PpCDC48II participated in the protection of gametophore cells that lead to the development of freezing tolerance. Previous studies of zebrafish CDC48 showed that the cold-inducible protein CDC48 may be regulated by transcription factors in response to cell proliferation and cell cycle progression under cold conditions [4]. Some evidences showed that CDC48 regulates cell cycle in various organisms, such as yeast, Arabidopsis, mouse, and human $[15,31,33,34]$. According to the results of cold-sensitive yeast mutants studies, cell division arrest occurs at the large budded cell stage, when there is an undivided nucleus and microtubule bundles spread throughout the cytoplasm from an unseparated spindle pole body [31]. Therefore, yeast CDC48 plays an important role in the $\mathrm{M}$ phase of cell cycle at low temperature, and these mutant phenotypes are consistent with defect in homotypic fusion of the inner nuclear and ER membranes.

Nevertheless, there are several reports describing that CDC48 has a variety of biological functions, including a role in regulating apoptosis [34-37]. CDC48p/p97/VCP is involved in a number of diverse cellular pathways through interactions with different adaptor proteins [38], which can distinguish between native and non-native proteins. Some results demonstrated $\mathrm{Cdc} 48 / \mathrm{VCP}$ to be crucially involved in several cellular functions controlled by ubiquitination [12]. The chaperone activity of CDC48 may be required to disassemble the SNARE (soluble N-ethylmaleimide-sensitive fusion attachment protein receptors) complex in membrane fusion, and to recognize proteins destined to be processed or degraded after their release from the ER [32]. In tobacco, some experiments indicated that $\mathrm{NgCDC} 48$ is involved in ER-associated degradation, and NgCDC48-mediated protein degradation, in turn, is required for normal growth and development of tobacco plants [19]. According to our previous study, differentially expressed ESTs analyzed under cold acclimation in $P$. patens, indicated that cold acclimation of $P$. patens reduced cell division and reprogrammed plant development, and the ubiquitin-proteasome system was proposed to be involved in this process [4]. Furthermore, our data demonstrated that disruption of PpCDC48II led to freezing hypersensitivity after cold acclimation, providing concrete evidence for the role of PpCDC48II as a positive regulator of cold-induced freezing tolerance in moss (Figure 3). These data also suggested that the acquisition of freezing tolerance in $P$. patens involves a $P p C D C 48 I I$-mediated signaling pathway. Therefore, we are tempted to consider that enhanced chaperone activity by $P p C D C 48 I I$ induction at cold temperatures may recognize and degrade misfolded proteins in gametophores. Higher levels of the CDC48 protein may be required to promote cellular functions in the cold than at normal growth temperature. We are currently conducting a yeast two-hybrid screen to identify PpCDC48II-interacting proteins and targeting experiment to define PpCDC48II localization. Further experiments are required to elucidate the mode of action of PpCDC48II and its interacting proteins.

The authors thank Dr. Mitsuyasu Hasebe for the kind gift of pTN182 vector. We are also very grateful to members of our laboratory for discussion and critical comments on the manuscript. This work was supported by the National Natural Science Foundation of China (Grant No. 30700404).

1 Reski R. Physcomitrella and Arabidopsis: the David and Goliath of reverse genetics. Trends Plant Sci, 1998, 3: 209-210

2 Schaefer D G. Gene targeting in Physcomitrella patens. CurrOpin Plant Biol, 2001, 4: 143-150

3 Anzu M, Manabu N, Keita A, et al. Abscisic acid-induced freezing tolerance in the moss Physcomitrella patens is accompanied by increased expression of stress-related genes. J Plant Physiol, 2003, 160: 475-483

4 Sun M M, Li L H, Xie H, et al. Differentially expressed genes under cold acclimation in Physcomitrella patens. J Biochem Mol Biol, 2007, 40: $986-1001$ 
5 Shintaro I, Nobuhiko O, Michiaki Y. Cold-inducible expression of the cell division cycle gene CDC48and its promotion of cell proliferation during cold acclimation in zebrafish cells. FEBS Lett, 2003, 549: 14-20

6 Hirsch C, Gauss R, Horn S C, et al. The ubiquitylation machinery of the endoplasmic reticulum. Nature, 2009, 458: 453-460

7 Ramadan K, Bruderer R, Spiga F M, et al. Cdc48/p97 promotes reformation of the nucleus by extracting the kinase Aurora B from chromatin. Nature, 2007, 450: 1258-1262

8 Halawani D, Latterich M. p97: The cell's molecular purgatory? Mol Cell, 2006, 22: 713-717

9 Janiesch P C, Kim J, Mouysset J, et al. The ubiquitin-selective chaperone CDC-48/p97 links myosin assembly to human myopathy. Nat Cell Biol, 2007, 9: 379-390

10 Alexandru G, Graumann J, Smith G T, et al. UBXD7 binds multiple ubiquitin ligases and implicates p97 in HIF1alpha turnover. Cell, 2008, 134: 804-816

11 DeBose-Boyd R A. Feedback regulation of cholesterol synthesis: sterol-accelerated ubiquitination and degradation of HMG CoA reductase. Cell Res, 2008, 18: 609-621

12 Ye Y. Diverse functions with a common regulator: ubiquitin takes command of an AAA ATPase. J Struct Biol, 2006, 156: 29-40

13 Sookhee P, David M R, Sebastian Y B. In planta analysis of the cell cycle-dependent localization of AtCDC48A and its critical roles in cell division, expansion, and differentiation. Plant Physiol, 2008, 148: 246-258

14 Rancour D M, Dickey C E, Park S, et al. Characterization of AtCDC48. Evidence for multiple membrane fusion mechanisms at the plane of cell division in plants. Plant Physiol, 2002, 130: 12411253

15 Feiler H S, Desprez T, Santoni V, et al. The higher plant Arabidopsis thaliana encodes a functional CDC48 homologue which is highly expressed in dividing and expanding cells. EMBO J,1995, 14: 5626-5637

16 Zimmermann P, Hirsch-Hoffmann M, Hennig L, et al. GENEVESTIGATOR: Arabidopsis microarray database and analysis toolbox. Plant Physiol, 2004, 136: 2621-2632

17 Zimmermann P, Hennig L, Gruissem W. Gene-expression analysis and network discovery using Genevestigator. Trends Plant Sci, 2005, 10: 407-409

18 Sookhee P, David M R, Sebastian Y B. In planta analysis of the cell cycle-dependent localization of AtCDC48A and its critical roles in cell division, expansion, and differentiation. Plant Physiol, 2008, 148: 246-258

19 Hansol B, Soo M C, Seong W Y, et al. Suppression of the ER-localized AAA ATPase NgCDC48 inhibits tobacco growth and development. Mol Cells, 2009, 28: 57-65

20 Ashton N W, Cove D J, Featherstone D R. The isolation and physiological analysis of mutants of the moss Physcomitrella patens. Planta, 1979, 144: 437-442

21 Minami A, Nagao M, Arakawa K, et al. Abscisic acid-induced freezing tolerance in the moss Physcomitrella patens is accompanied by increased expression of stress-related genes. J Plant Physiol, 2003, 160: 475-483

22 Porra R J, Thompson W A, Kriedemann P E. Determination of accu- rate extinction coefficients and simultaneous equations for assaying chlorophylls $\mathrm{a}$ and $\mathrm{b}$ extracted with four different solvents: verification of the concentration of chlorophyll standards by atomic absorption spectroscopy. Biochimica et Biophysica Acta, 1989, 975: 384-394

23 Nishiyama T, Fujita T, Shin I T, et al. Comparative genomics of Physcomitrella patens gametophytic transcriptome and Arabidopsis thaliana: Implication for land plant evolution. Proc Natl Acad Sci USA, 2003, 100: 8007-8012

24 Pearce R S. Molecular analysis of acclimation to cold. Plant Growth Regul, 1999, 29: 47-76

25 Thomashow M F. Plant cold acclimation: freezing tolerance genes and regulatory mechanisms. Annu Rev Plant Physiol Plant Mol Biol, 1999, 50: 571-599

26 Xin Z, Browse J. Cold comfort farm: the acclimation of plants to freezing temperatures. Plant Cell Environ, 2000, 23: 893-902

27 Seki M, Narusaka M, Abe $\mathrm{H}$, et al. Monitoring the expression pattern of 1300 Arabidopsis genes under drought and cold stresses by using a full-length cDNA microarray. Plant Cell, 2001, 13: 61-72

28 Anzu M, Manabu N, Keiichi I, et al. Cold acclimation in bryophytes: low-temperature-induced freezing tolerance in Physcomitrella patens is associated with increases in expression levels of stress-related genes but not with increase in level of endogenous abscisic acid. Planta, 2005, 220: 414-423

29 Dong C N, Danyluk J, Wilson K E, et al. Cold-regulated cereal chloroplast late embryogenesis abundant-like proteins. Molecular characterization and functional analyses. Plant Physiol, 2002, 129: 13681381

30 Hara M, Terashima S, Fukaya T, et al. Enhancement of cold tolerance and inhibition of lipid peroxidation by citrus dihedron in transgenic tobacco. Planta, 2003, 217: 290-298

31 Fröhlich K U, Fries H W, Rudiger M, et al. Yeast cell cycle protein CDC48p shows full-length homology to the mammalian protein VCP and is a member of a protein family ivolved in secretion, peroxisome formation, and gene expression. J Cell Biol, 1991, 114: 443-453

32 Petek B, Aysun P, Fadime A K. Different p97/VCP complexes function in retrotranslocation step of mammalian Er-associated degradation (ERAD). Int J Biochem Cell Biol, 2011, 43: 613-622

33 Shirogane T, Fukada T, Muller J M, et al. Synergistic roles for Pim-1 and c-Myc in STAT3-mediated cell cycle progression and antiapoptosis. Immunity, 1999, 11: 709-719

34 Müller J M, Deinhardt K, Rosewell L, et al. Targeted deletion of p97 (VCP/CDC48) in mouse results in early embryonic lethality. BBRC, 2007, 354: 459-465

$35 \mathrm{Wu} \mathrm{D}$, Chen P J, Chen S, et al. C. elegans MAC-1, an essential member of the AAA family of ATPases, can bind CED-4 and prevent cell death. Development, 1999, 126: 2021-2031

36 Ralf J B, Hans Z. Mechanisms of Cdc48/VCP-mediated cell deathfrom yeast apoptosis to human disease. Biochim Biophys Acta, 2008, 1783: 1418-1435

37 Rati V, Robert O, Ruihua F, et al. Cdc48/p97 mediates UV-dependent turnover of RNA Pol II. Mol Cell, 2010, 41: 82-92

38 Yeung $\mathrm{H}$ O, Kloppsteck P, Niwa $\mathrm{H}$, et al. Insights into adaptor binding to the AAA protein p97. Biochem Soc Trans, 2008, 36: 62-72

Open Access This article is distributed under the terms of the Creative Commons Attribution License which permits any use, distribution, and reproduction in any medium, provided the original author(s) and source are credited. 